\title{
Indoor localization using NFC and mobile sensor data corrected using neural net
}

\author{
Tibor Tajti, Gábor Geda, Tamás Balla, Gyula Vad \\ Eszterhazy Karoly University of Applied Sciences \\ tajti@aries.ektf.hu, gedag@aries.ektf.hu, ballatamas92@gmail.com, \\ gyulavad@gmail.com
}

\begin{abstract}
We are researching the RFID/NFC technology combined with other possibilities available in mobile phones. We are trying to use mobile sensors and NFC together for indoor positioning. Localization and navigation is a fast emerging area, we are using it when driving our car or to find a shop or other target in near. It is possible with the help of the GPS (Global Positioning System), but it is not working indoors. Fortunately mobile phones have many possibilities which can be used for localization in such environments. They can measure signal strengths of WIFI or Bluetooth stations. Some indoor localization projects are based on that method. In our project we try to get the best result from the possibilities of several sensors available in mobile phone. Such sensors are: magnetometer, gravitation, acceleration and gyroscope sensors. There are virtual sensors (eg.:linear acceleration) which are computed values derived from the other ones. The sensors provide enough information to compute the direction, speed and then the position, but their values are noisy, so the cumulated error grows with the time. Fortunately we have some possibilities to deal with the error. We can combine redundant information to reduce the error, and we can use reference positions thanks to the NFC (Near Field Communication) technology. More and more mobile phones have NFC chips, which are easily able to get information from NFC tags. Such NFC tags are not expensive, they can be placed on the walls and querying them takes very little time. QR (Quick Response) code can be a backup solution, which can be queried using the mobile phones camera. NFC or QR reference positions are very simple and effective solution to get the exact location when the computed position's error is too large. Other good possibility of the mobile phones is their communication and computation ability. The data collected from the sensors can be stored in a database where more intelligent algorithms can be tried in addition to the simple computations described above. We try to teach neural network algorithm to give good outputs from the sensor data including the virtual sensors and our computed values.
\end{abstract}

Keywords: NFC, hybrid technology, sensor data, mobile, localization, neural 
network

$M S C: 68 \mathrm{~N} 01,68 \mathrm{~T} 05$

\section{Introduction}

Most of the smart mobile phones have GPS receiver, which allows the outdoor positioning accuracy of a few meters. This technology depends on the usability of the GPS satellite signals received. This may be blocked by objects (mountains, trees, buildings) in the area, and is usually unuseful indoors. In such cases other positioning techniques can be used, for example: when GSM , WIFI or Bluetooth network signals are available, we can use signal strength for calculation of the distance from the transmitter (RSSI - Received Signal Strength Indication). We also can use information from mobile device's motion sensor data. In buildings, caves, basement garage, where GPS providers may be unavailable, however, it would be useful to have the possibility of positioning services. The importance of the task indicated that the manufacturers have created an organization called In-Location Alliance.[1]

\section{Related work}

The In-Location Alliance established in August of 2012. More than twenty companies participate in this organization, they work together and share their experiences on pre-commercial pilots. In this project they use the signal strength of nearest Wi-Fi providers and the mobile device's built in sensors to get accurate, realtime localization where GPS providers are unavailable or noisy.[1] In a different project the researchers examined different method for RSSI based indoor localization. They tried to combine the RSSI based methods with Aritfical Neural Networks for more accurate results. During their research they found that the backpropagation algorithm is more accurate than probabilistic model. In the future they plan optimizing the ANN by genetic algorithm and they hope, they can achieve more useful result.[5] On the other hand other researcher try to use mobile sensors to locate a device or a people inside a building. The programmers who participate in this type of researches developed applications to collect data from the mobile sensors. They use the collected data for sensor based indoor localization. The most important advantages of sensors based indoor localization that it does not need any external infrastructure.[3]

\section{Measurement method}

Our tests were examined using a few mobile phones of different types. We have developed one application on the Android platform. The application has several 
features and parameters which are useful for our experiments. In case where multiple sensors or virtual sensors are available for the same purpose in the application we can switch between them. The sensor reading frequency and the step length can be set as well. For the reference points three ways can be used in the application. Reference points can be read from NFC tags, this is the easiest and fastest way. In case our mobile does not have NFC chip, the QR code can work for us using the camera and a built-in QR reader library. For testing we have also used reference point parameters, which can be set in the settings dialogue. Several different tests have been examined, in most of them we had 4 reference points. Shorter tests (below 1 minute) were sufficient to test our application, longer tests have produced enough data for teaching neural network. All the tests produced the measured sensor data in text files with comma separated values, and also uploaded into a relational database, so we were easily able to run data analysis on these data.

\section{Development on data analysis and calculations}

Our goal is to develop mobile application for indoor positioning. Mobile development is a slow process, building and deploying the application can take several minutes. So after we have developed the data gathering and uploading mobile application, the next development steps are first to be done in a very productive environment, where the algorithms can be run instantly. One such environment is the Python programming language with array operations, easy to use graphing and several available neural network algorithms. The algorithms can be also run in IPython Notebook, which helps the collaboration between team members.

\section{Position calculations}

We use the direction and acceleration sensor data to calculate the position of the device. From the direction and acceleration data we can compute the speed vector, then from the speed vector we can get the position.

Since the sensor data noise and the caused calculated position error will be to large, so reliable reference points will be used. Reference points can be read from NFC tags, QR codes, calculated from WIFI signal strength.

By now we only could make tests with few reference points. Since the neural network need lot of training data, we transformed the positions between the reference points to match them. So we have many training points, although there can be errors in them.

\section{Data analysis and filtering}

We have collected data from several physical or virtual mobile sensors and analysed them. Now take a look at the most important ones. One major data is the direction of the mobile phone. This sensor has 3 dimensions, we will now see the differences 
in the y coordinate. Figure 1 shows that the direction value has continuously some low level noise and occasionally higher as well. This noise can come both from the device and from the human hand. The device's own noise was about 1 magnitude smaller than the device's sensor noise plus the human hand.

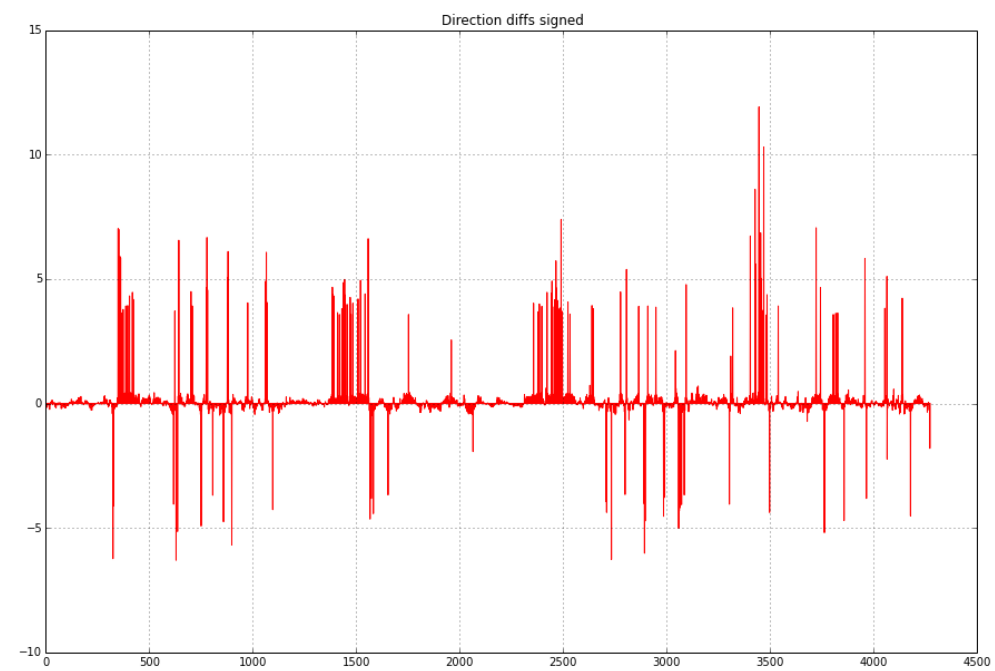

Figure 1: Difference from previous direction (Y coordinate)

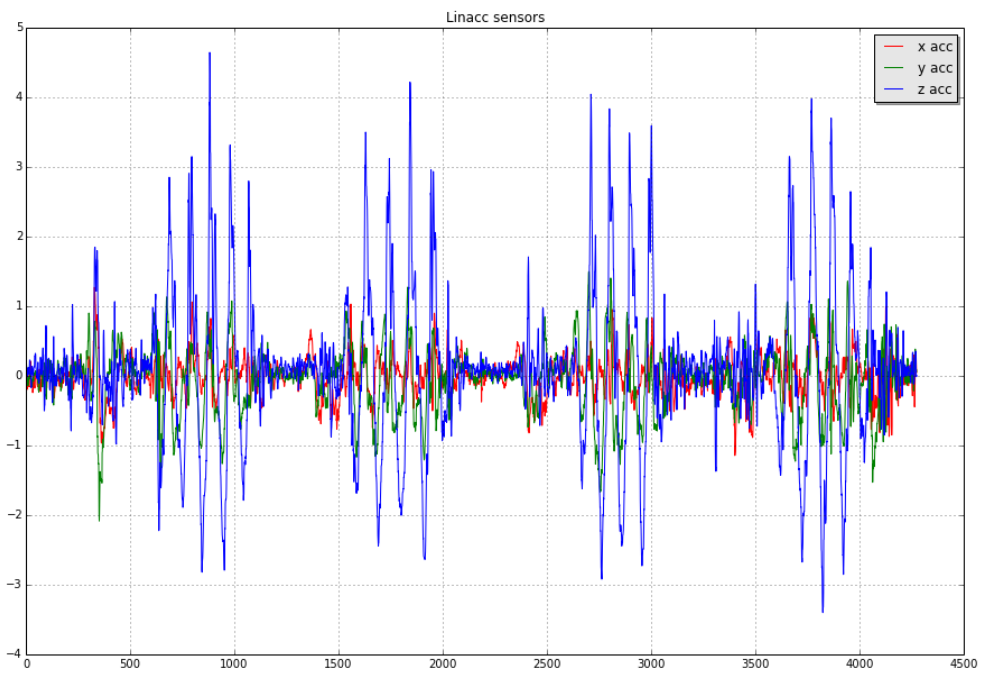

Figure 2: Linear acceleration sensor data

Figure 2 shows the linear acceleration sensor data, all 3 coordinates. Some noise 
seems to be there and we can see that the $\mathrm{Z}$ coordinate has the highest movements. This is because the human steps. We can use this data for step counting, which is one way to correct our noisy data.

Since the $\mathrm{Z}$ direction is only important for the step counting there the noise is not a big problem. So we take focus on the $\mathrm{X}$ and $\mathrm{Y}$ coordinates. Both of them are very noisy and very important. The error in acceleration data will occur cumulated in the calculated speed data, and then in the calculated position data. Figure 3 shows that the sensor error data causes large error in the calculated position. The test happened in a square with side length of $3 \mathrm{~m}$.

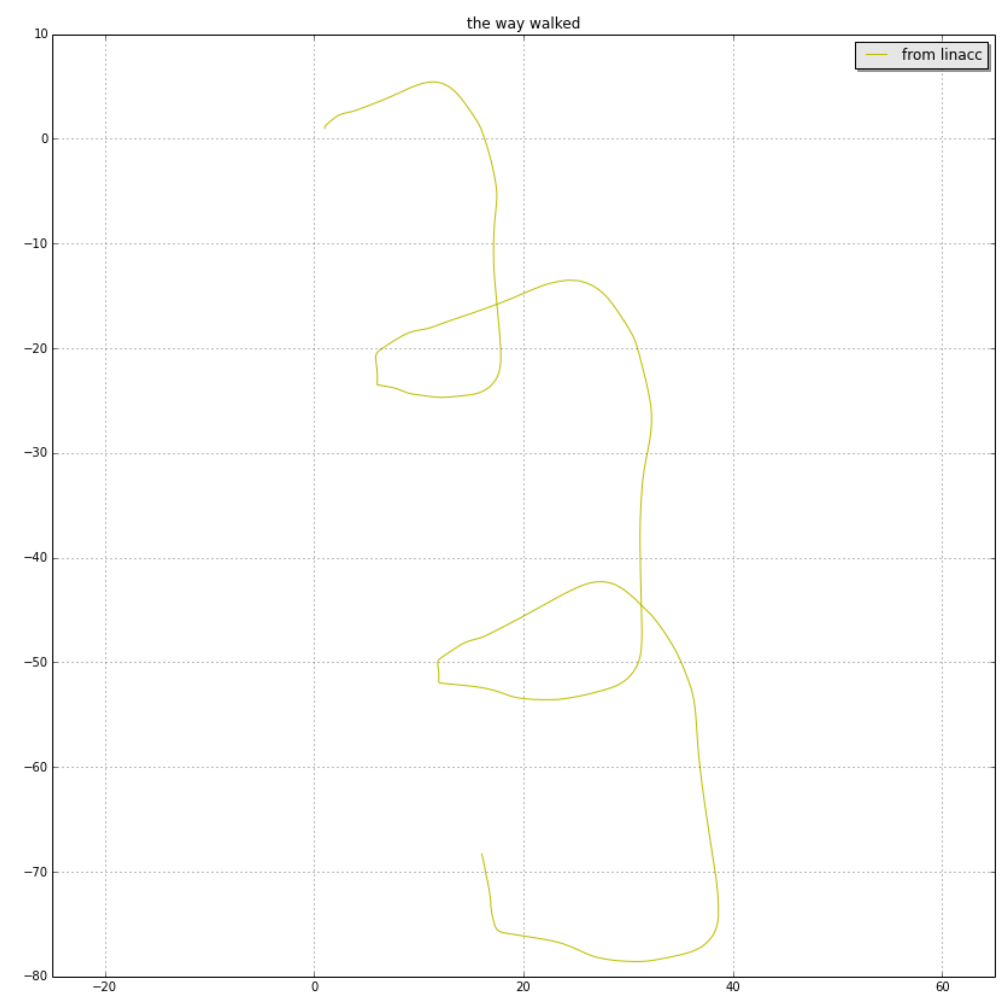

Figure 3: Position calculated from linear acceleration sensor data

So we see that the cumulated error is unacceptable. Noise filtering can help a little bit. The unfiltered sensor data is as shown on the Figure 4. 


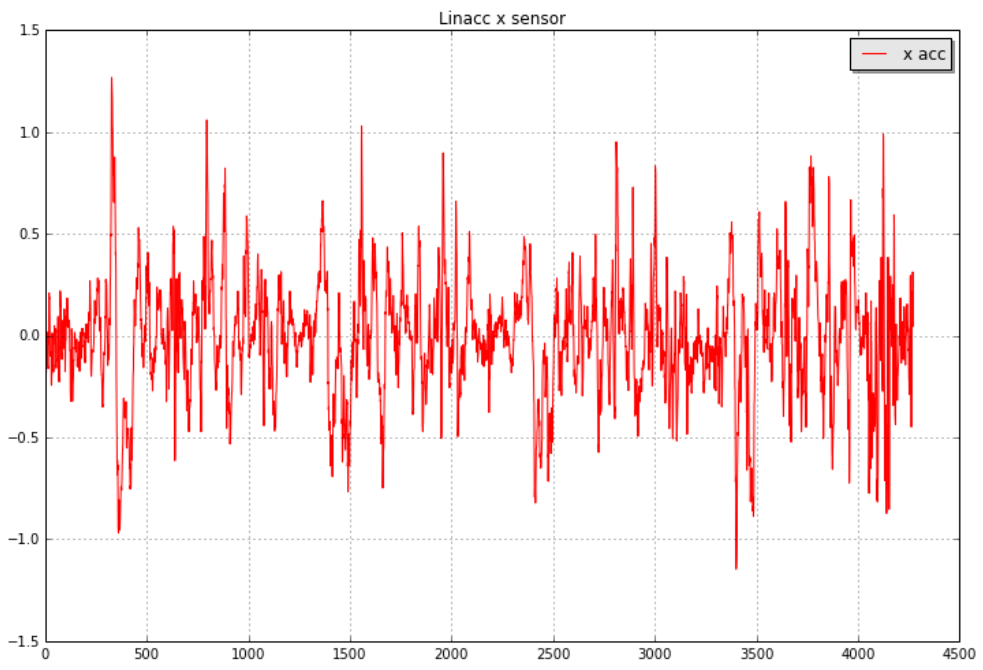

Figure 4: Linear acceleration sensor $\mathrm{X}$ coordinate

The noise can be reduced, several algorithms are available for that. One simple noise filtering produced the result shown on Figure 5.

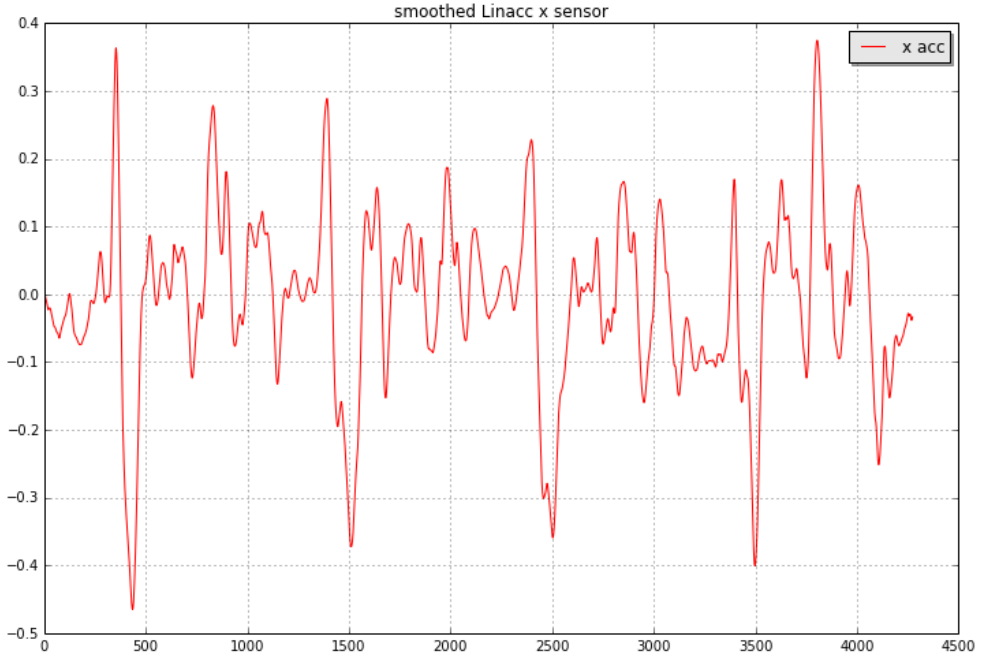

Figure 5: Linear acceleration sensor X coordinate after noise filtering 


\section{Neural network}

Neural networks provide the possibility to learn the patterns in the training data and use them for other data of the same kind. We have built the architecture to provide sensor data for the neural network as input and the target acceleration data as the output. These target acceleration data values were calculated so that they will lead to the real positions.

\section{Conclusions}

The neural network in our case was able to learn to predict acceleration data which may lead to better position calculation. Our next task is to test this.

\section{References}

[1] http://www.in-location-allianz.com/

[2] Shay Reuveny, Maayan Zadik: 3D Motion tracking with Gyroscope and Accelerometer

[3] https://github.com/hfalan/sensor-log-android

[4] http://en.wikipedia.org/wiki/Indoor_positioning_system

[5] Hamid Mehmood, Nitin K. Tripathi and Taravudh Tipdecho: Indoor Positioning System Using Artificial Neural Network 